\title{
Variación Diurna de la Talla en una Población de Adultos Jóvenes Colombianos
}

\author{
Diurnal Variation of Height in a Colombian Young Adult Population
}

\author{
Andrés Felipe Chaparro*; Gabriel David Pinilla*; Juan Sebastián Arias* \& Luis Ernesto Ballesteros ${ }^{* *}$
}

CHAPARRO, A. F.; PINILLA, G. D; ARIAS, J. S. \& BALLESTEROS, L. E. Variación diurna de la talla en una población de adultos jóvenes colombianos. Int. J. Morphol., 32(4):1365-1369, 2014.

RESUMEN: Los cambios en la talla corporal a lo largo del día tras la bipedestación son procesos poco conocidos, aun en el escenario de la morfología. Este estudio evaluó la variación de la talla a lo largo del día en una muestra de población joven colombiana. Mediante un muestreo aleatorio estratificado por asignación proporcional se evaluó la variación de la talla corporal a lo largo del día en 55 sujetos jóvenes. Se tomaron cuatro mediciones de talla durante un día, usando para ello un tallímetro convencional. Se realizó análisis estadístico de los resultados mediante pruebas de concordancia (CCI). La edad promedio de los sujetos evaluados fue de 19,5 $\pm 1,86$ años, de los cuales 22 (40\%) fueron del sexo masculino. La talla y peso promedio fueron $167,1 \pm 8,59 \mathrm{~cm}$ y $65,8 \pm 14,5 \mathrm{Kg}$, respectivamente. La pérdida media de altura al final del día fue $9,4 \pm 3,12 \mathrm{~mm}$; la pérdida en las mujeres fue de $9,72 \pm 3,35 \mathrm{~mm}$ y en los hombres $8,95 \pm 2,76 \mathrm{~mm}$ sin que esa diferencia fuese significativa $(\mathrm{p}=0,22)$. No se identificó asociación entre peso mayor a $78 \mathrm{Kg}$ y pérdida de talla en el día mayor a $1 \mathrm{~cm}(\mathrm{p}=0,18)$. En la franja de 06:00-10:00 horas ocurrió la mayor disminución de la talla, el 60\%; mientras que de 10:00-14:00 horas la disminución fue 10\%, y del 30\% de 14:00-18:00 horas. La pérdida de talla observada en este estudio es ligeramente inferior a lo reportado en estudios previos. El conocimiento de la variación diaria de la talla corporal debe tenerse en cuenta en el planeamiento de los controles de crecimiento infantil.

PAlABRAS ClAVE: Altura, Peso; Ciclos circadianos; Crecimiento; Adulto; Colombia.

\section{INTRODUCTION}

El cambio en la medida de los tejidos es un determinante de la talla en el ser humano, correspondiendo a los discos intervertebrales, que están sometidos a diversas fuerzas, como la ejercida por la gravedad, evidenciable tras la bipedestación, el mayor compromiso en esta variabilidad (Baker et al., 1978; Buckler, 1978; Tyrrell, 1985; Moore et al., 2011). Los discos intervertebrales (DI) ubicados entre los cuerpos de las vértebras cervicales, torácicas y lumbares, proporcionan una adhesión potente entre estas. Los DI conforman el 20-25\% de la longitud total de la columna vertebral (Franquel \& Nordin, 2001; Panjabi, 2003; Kapandji, 2012). Cada DI está configurado por dos estructuras: un núcleo pulposo compuesto por proteoglicanos, colágeno y 60-90\% de agua; y un anillo fibroso de láminas concéntricas de fibrocartílago y proteoglicanos que otorga dureza y estabilidad al disco (Park, 1997; Rouviére \& Delmas, 1999).

La compresión mecánica sobre la región axial del cuerpo, evidente durante la bipedestación, genera que el agua que posee el núcleo pulposo migre hacia el exterior, deshidratándolo y provocando así una perdida en su volumen, que se refleja en la talla del individuo (Park). Por el contrario, cuando el individuo se encuentra en decúbito (prono, supino o lateral), el efecto de la gravedad sobre los tejidos de referencia se torna nula. La hidrofilia que le brindan los proteoglicanos al núcleo pulposo genera un flujo de agua hacia el interior de este, que le permite recuperar su hidratación y su volumen (Williams et al., 1998).

La variación de la talla corporal a lo largo del día ha sido reportada en un rango de $0,98-1,93 \mathrm{~cm}$ existiendo consenso en que el mayor porcentaje de esta pérdida se observa en las primeras horas de inicio de la actividad diaria (Reilly et al., 1984; Lampl et al., 1992; Ledsome et al., 1996; Tillman \& Clayton, 2001). Por otro lado, se ha reportado que el $71 \%$ de la estatura perdida es recuperada en la primera mitad del sueño.

\footnotetext{
* Estudiante Escuela Medicina. Universidad Industrial de Santander, Bucaramanga, Colombia.

${ }^{*}$ MD, MSc. Profesor titular. Universidad Industrial de Santander, Bucaramanga, Colombia.
} 
El comportamiento de la variación diaria de la talla corporal y del volumen de los discos intervertebrales ha sido descrito en otros grupos poblacionales. La ausencia de este tipo de información en población mestiza colombiana y el relativo amplio rango de esta variabilidad reportado en estudios previos, le da pertinencia a la realización del presente estudio, con el propósito de enriquecer la información sobre este evento morfo fisiológico, que a su vez derive en adecuadas aplicaciones médicas.

\section{MATERIAL Y MÉTODO}

Se realizó un estudio cross-sectional con muestreo aleatorio estratificado de asignación proporcional en una población de 112 adultos jóvenes. Para el objetivo descriptivo, el cálculo de la muestra se realizó con la aplicación OpenEpi® (Sullivan et al., 2009), obteniéndose una muestra de 55, con un intervalo de confianza mayor al 99,99\%. Para garantizar la veracidad de las mediciones, previo al inicio del proyecto, se realizó un taller de estandarización y entrenamiento de los examinadores, donde se establecieron las técnicas y parámetros de medición.

Dos evaluadores de manera independiente realizaron mediciones de la talla corporal a cada uno de los individuos evaluados, cuatro veces al día, con un tallímetro convencional, en los horarios de las 06:00, 10:00, 12:00, y 18:00 horas (Fig. 1). Igualmente, de manera simultánea a la primera y la última evaluación se tomó el peso corporal con una báscula digital (Kenwell@). Los datos obtenidos por ambos examinadores fueron tabulados por un tercer investigador en el software Microsoft Excel®. De esta manera se desarrolló un triple enmascaramiento, donde ni los dos examinadores ni el tabulador conocían los resultados de las mediciones.

La concordancia de las mediciones realizadas por los dos examinadores fue evaluada mediante la prueba de Coeficiente de Correlación Intercalase. Se describieron las variables de importancia sociodemográficas, biológicas y clínicas encontradas en el grupo evaluado. Además, mediante el uso de razones de prevalencia, se identificaron variables asociadas con significancia estadística que indujeran al cambio de la talla corporal durante el día.
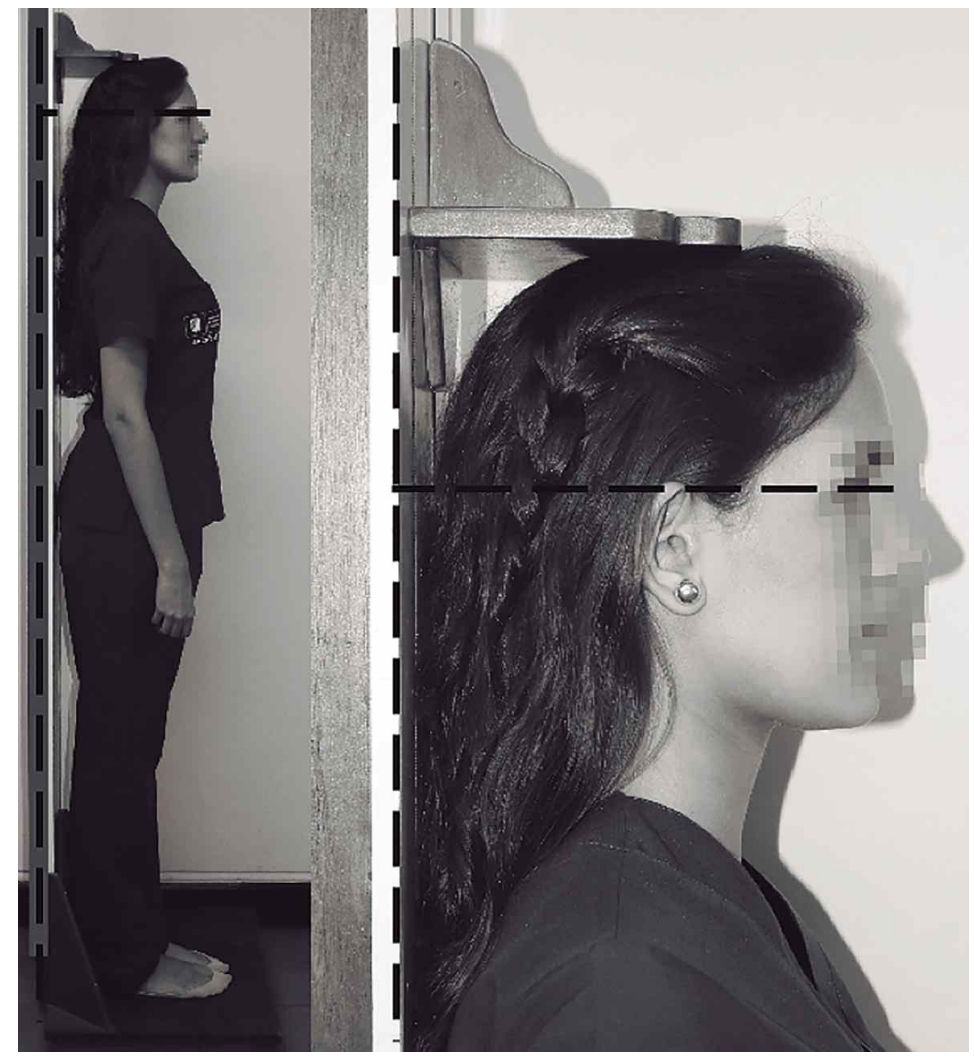

Fig. 1. Tallímetro y posición para medición.

\section{RESULTADOS}

En el estudio se midieron 55 adultos jóvenes, 22 hombres (40\%) y 33 mujeres, con una edad media de 19,5 $\pm 1,86$ años, quienes durante el día de las mediciones estuvieron normalmente activos. Los sujetos evaluados, tenían una altura media de $167,1 \pm 8,59 \mathrm{~cm}$ y un peso de $65,8 \pm 14,5 \mathrm{Kg}$. La pérdida total de la talla en el transcurso del día, fue de $9,4 \pm 3,12 \mathrm{~mm}$ correspondiendo a hombres $8,95 \pm 2,76 \mathrm{~mm}$ y mujeres $9,72 \pm 3,35 \mathrm{~mm}$, no se identificó diferencias estadísticamente significativas (RP 0,92 -IC95\% 0,46-1,85-; p=0,22.) (Fig. 2). La pérdida de la talla corresponde al $1,5 \%$ de la talla total.

En las primeras cuatro horas de medición (06:00-10:00 am) se observó perdida de $5,37 \pm 2,22 \mathrm{~mm}$, un $60 \%$ de la pérdida total diaria. En la franja de 10:00-14:00 horas la perdida de talla fue de 2,09 $\pm 2,72$ $\mathrm{mm}$, un $10 \%$ del total, mientras que la perdida en el intervalo de las 14:00-18:00 horas fue de 2,53 $\pm 2,93 \mathrm{~mm}$, un 30\% del total. Estas pérdidas fueron similares en hombres y mujeres (Fig. 3).

En relación a la variabilidad de las mediciones, se aplicó el Coeficiente de Correlación Intraclase (CCI) para las diferentes medidas, obteniéndose para las cuatro mediciones realizadas durante el día un CCI >0,99; sin diferencias estadísticamente significativas en las 


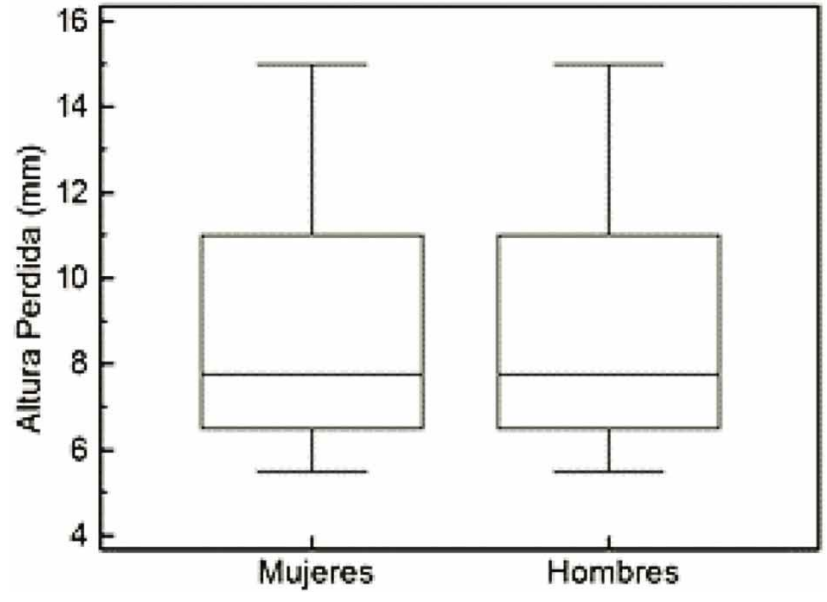

Fig. 2. Pérdida de talla corporal total (mm) según sexo.

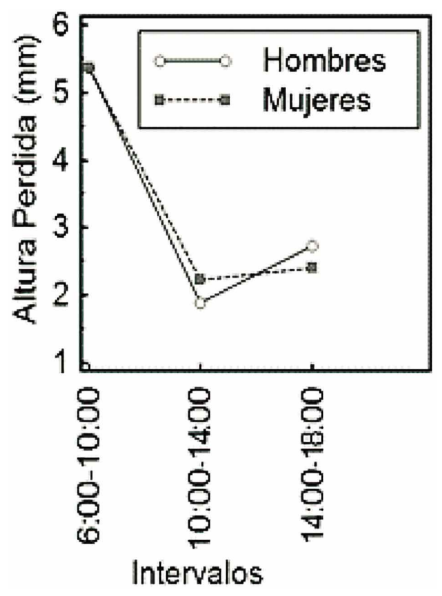

Fig. 3. Pérdida de talla corporal (mm) según sexo e intervalos de mediciones.

mediciones realizadas por los dos observadores, de manera independiente, pero concurrente. Se evaluaron otras variables obteniéndose diferentes valores de asociación con una pérdida de altura mayor a $1 \mathrm{~cm}$ sin que mostraran relación estadísticamente significativa: peso $>78 \mathrm{Kg}$ (RP 1,38 IC95\% 0,67-2,86; p=0,18) (Fig. 4); altura $>1,70 \mathrm{~m}(1,65$ IC95\% $0,78-3,49 ; \mathrm{p}=0,14)$; horas de sueño $>6$ (RP $1,71-\mathrm{IC} 95 \%$ $0,77-3,80 ; \mathrm{p}=0,12)$; horas desde suspensión del reposo $<1$ (RP 1,91-IC95\% 0,77-4,74; $p=0,10)$; $y$ edad $>19$ años (RP 1,00-IC95\% 0,50-1,99; $\mathrm{p}=0,22)$.

\section{DISCUSIÓN}

La variación diaria de la talla corporal ha sido escasamente descrita en diferentes grupos poblacionales y con

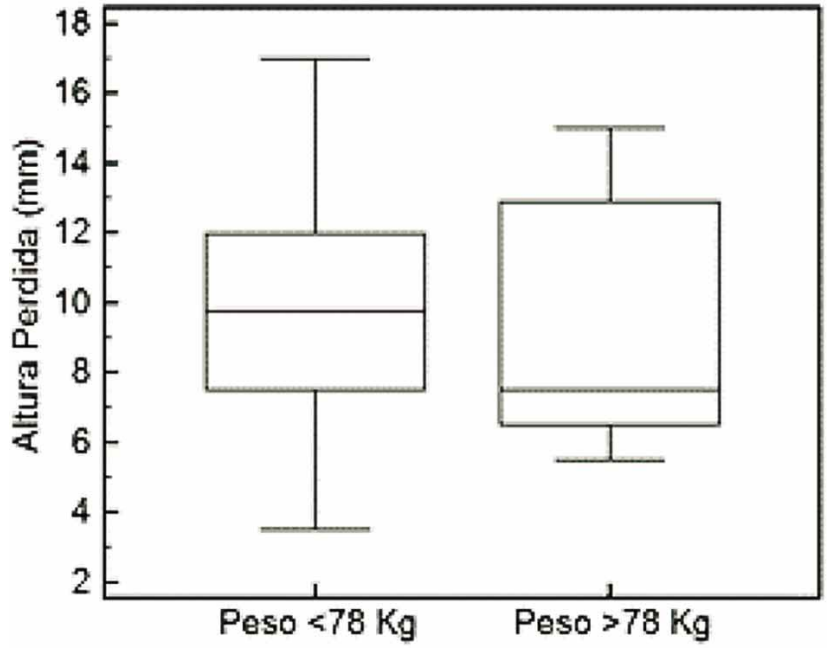

Fig. 4. Pérdida de talla corporal total $(\mathrm{cm})$ según peso.

resultados variables. Los estudios previos fueron realizados en poblaciones de raza caucásica, en EE.UU. (Tillman \& Clayton; Lampl), Canadá (Bostford et al., 1994), e Inglaterra (Reilly et al.), mientras que Park, evaluó esta variación de la talla en un población mongólica residente en Corea. Nuestro estudio es el primero en realizarse en el área Latinoamericana.

La pérdida de la talla corporal a lo largo del día observada en nuestro estudio $(0,94 \mathrm{~cm})$ es concordante con el reporte de Lampl $(0,98 \mathrm{~cm})$, situándose en el rango inferior de lo reportado en la literatura. Tillman \& Clayton, reportan un valor intermedio de $1,44 \mathrm{~cm}$. La máxima perdida de talla corporal $(1,93 \mathrm{~cm})$ ha sido reportada por Reilly et al., quien realizo nueve mediciones diarias en ocho sujetos adultos. Se resalta que los valores obtenidos en el estudio de Lampl, fueron tomados de un adolecente durante el periodo de un año con dos mediciones diarias. Otros estudios, utilizando técnicas de resonancia nuclear han reportado perdida diaria de volumen de los discos intervertebrales en un rango de 14,5-16,2\% (Botsford et al.; Park).

Tillman \& Clayton, reportaron una mayor pérdida de estatura en la primeras tres horas del día $(0,94 \mathrm{~cm})$, igualmente Reilly et al. Reporta una perdida en esta franja de $1,54 \mathrm{~cm}(80 \%)$. Nuestro estudio reporta una mayor perdida en las primeras cuatro horas del día (60\%), siendo estos hallazgos concordantes con el concepto de mayor perdida en las primeras horas del día, pero con un porcentaje ligeramente menor con lo reportado en estudios previos.

Encontramos en nuestro estudio una pérdida de la talla de $1,5 \%$ en individuos con una talla media de $167,1 \mathrm{~cm}$. Este valor se encuentra ligeramente por encima de lo señalado por Reilly et al. $(1,1 \%)$, en sujetos con un talla de 175 
$\mathrm{cm}$. No es posible justificar la diferencia entre estos dos estudios debido a que no encontramos significancia estadística para una pérdida de altura mayor a $1 \mathrm{~cm}$ con una que supere $170 \mathrm{~cm}$. Para poder descartar esta relación se requiere realizar un estudio que alcance un mayor poder estadístico. De igual forma, no es posible comparar los resultados obtenidos en este estudio con los obtenidos en los trabajos previos que utilizaron resonancia magnética nuclear, por obvias diferencias metodológicas.

Los estudios previos han realizado un abordaje netamente descriptivo por lo que se desconoce la asociación entre la magnitud de la pérdida de la talla con relación a variables independientes tales como el peso, edad y sexo. En este estudio no se identificó asociación entre un peso $>78 \mathrm{Kg}$ y perdida de talla al día $>1 \mathrm{~cm}(\mathrm{p}=0,18)$, esto a pesar de que bajo una óptica biofísica, un valor mayor de la masa corporal conlleva a un peso gravitatorio mayor, lo cual favorecería un aumento en la presión sobre los discos intervertebrales, con su consecuente deshidratación temprana. A su vez, no se identificaron diferencias estadísticamente significativas por género $(\mathrm{p}=0,62)$. Se destaca que es el primer estudio donde hacen parte de la muestra individuos del sexo femenino y donde el total de individuos analizados es mayor a 10.

La variación de talla del individuo, cobra importancia en la elaboración de las metodologías asociadas a los programa de control de crecimiento y desarrollo y diagnóstico de patologías relacionadas, especialmente en escolares y adolescentes (Buckler).

Finalmente, llama la atención la consideración que los autores citados en este material, hacen de la variación de la talla a lo largo del día, como un fenómeno circadiano. Ante esto, es necesario considerar otro tipo de variables probablemente endocrinas (bioquímicas, cuantitativas), que permitan determinar la influencia de los ciclos de vigilia y sueño, no como periodos de descanso, sino como interacción entre el ambiente y la autorregulación neuroendocrina (Krystal et al., 2013).

CHAPARRO, A. F.; PINILLA, G. D; ARIAS, J. S. \& BALLESTEROS, L. E. Diurnal variation of height in a Colombian young adult population. Int. J. Morphol., 32(4):1365-1369, 2014.

SUMMARY: Changes in height throughout the day after standing are poorly understood, even in morphological sciences. This study evaluated height variations throughout the day in a sample of young Colombian population. Using a stratified random sampling with proportional allocation, diurnal changes in height was evaluated in 55 subjects. Four measurements of size were taken over a day, using a conventional measurer for it. Statistical analysis of the results was performed by testing agreement (ICC). The average age of the subjects tested was $19.5 \pm 1.86$ years, of which $22(40 \%)$ were male. The average height and weight were $167.1 \pm 8.59 \mathrm{~cm}$ and $65.8 \pm 14.5$ $\mathrm{Kg}$ respectively. The average loss of height after the day was $9.4 \pm 3.12 \mathrm{~mm}$ loss in women was $9.72 \pm 3.35 \mathrm{~mm}$ and $8.95 \pm 2.76 \mathrm{~mm}$ in men but this difference was not significant $(\mathrm{p}=0.22)$. Statistically significant association between weight $>78 \mathrm{Kg}$ and height loss in the day $>1 \mathrm{~cm}(\mathrm{p}=0.18)$ was not identified. In the range of 06:00 to 10:00 hours came up with the greatest reduction in size, $60 \%$; while from 10:00 to 14:00 hours the decrease was $10 \%$ and 30\% of 14:00-18:00 hours. Height loss observed in this study was just below that reported in the literature. The knowledge of the changes in height throughout the day should be taken in consideration when diagnosing growth pathologies.

KEY WORDS: Height; Weight; Circadian cycles; Growth; Adult; Colombia.

\section{REFERENCIAS BIBLIOGRÁFICAS}

Baker, I. A.; Hughes, J. \& Jones, M. Temporal variation in the height of children during the day. Lancet, 1(8077):1320, 1978.

Botsford, D. J.; Esses, S. I. \& Ogilvie-Harris, D. J. In vivo diurnal variation in intervertebral disc volume and morphology. Spine (Phila Pa 1976), 19(8):935-40, 1994.

Buckler, J. M. Variations in height throughout the day. Arch. Dis. Child, 53(9):762, 1978.

Franquel, V. H. \& Nordin, M. Biomecánica básica del sistema musculoesquelético. $3^{\mathrm{a}}$ ed. Madrid, McGraw Hill Interamericana, 2001.
Kapandji, A. I. Fisiología articular, Tronco y Raquis. $6^{\mathrm{a}}$ ed. Madrid, Médica Panamericana, 2012.

Krystal, A. D.; Benca, R. M. \& Kilduff, T. S. Understanding the sleep-wake cycle: sleep, insomnia, and the orexin system. $J$. Clin. Psychiatry, 74(Suppl. 1):3-20, 2013.

Lampl, M. Further observations on diurnal variation in standing height. Ann. Hum. Biol, 19(1):87-90, 1992.

Ledsome, J. R.; Lessoway, V.; Susak, L. E.; Gagnon, F. A.; Gagnon, R. \& Wing, P. C. Diurnal changes in lumbar intervertebral distance, measured using ultrasound. Spine (Phila Pa 1976), 


$$
\text { 21(14):1671-5, } 1996 .
$$

Moore K. L.; Dalley A. F. \& Agur, A. M. R. Clinically Oriented Anatomy. 6th ed. Philadelphia, Wolters Kluwer/Lippincott Williams \& Wilkins, 2010.

Panjabi, M. M. Clinical spinal instability and low back pain. $J$. Electromyogr. Kinesiol., 13(4):371-9, 2003.

Park, C. O. Diurnal variation in lumbar MRI. Diurnal variation in lumbar MRI. Correlation between signal intensity, disc height, and disc bulge. Yonsei Med. J., 38(1):8-18, 1997.

Reilly, T.; Tyrrel, A. \& Troup, J. D. Circadian variation in human stature. Chronobiol. Int., 1(2):121-6, 1984.

Rouviére, H. \& Delmas, A. Anatomía Humana. Descriptiva, Topográfica y Funcional. 10ª ed. Barcelona, Masson, 1999.

Sullivan, K. M.; Dean, A. \& Soe M. M. OpenEpi: a web-based epidemiologic and statistical calculator for public health. Public Health Rep., 124(3):471-4, 2009

Tillmann, V. \& Clayton, P. E. Diurnal variation in height and the reliability of height measurements using stretched and unstretched techniques in the evaluation of short-term growth. Ann. Hum. Biol., 28(2):195-206, 2001.

Tyrrell, A. R.; Reilly, T. \& Troup, J. D. Circadian variation in stature and the effects of spinal loading. Spine (Phila Pa 1976), 10(2):161-4, 1985.

Williams, P. L.; Bannister, L. H.; Berry, M. M.; Collins, P.; Dyson, M.; Dussek, J. E. \& Ferguson, M. W. J. Anatomía de Gray. Bases Anatómicas de la Medicina y la Cirugía. $38^{\mathrm{a}}$ ed. Madrid, Churchill Livingstone-Harcourt Brace, 1998.

\section{Dirección para Correspondencia: \\ Dr. Luis Ernesto Ballesteros A. \\ Avenida González valencia \#54.25 Apto 404. \\ Bucaramanga \\ COLOMBIA}

Email: Iballest56@yahoo.es

Recibido : 18-02-2014

Aceptado: 15-07-2014 